Pacific

Journal of

Mathematics

\title{
RATIONAL SEIFERT SURFACES \\ IN SEIFERT FIBERED SPACES
}

JoAn E. LiCATA AND JoshuA M. SABLOFF 


\title{
RATIONAL SEIFERT SURFACES IN SEIFERT FIBERED SPACES
}

\author{
JOAN E. LICATA AND JOSHUA M. SABLOFF
}

\begin{abstract}
Rationally null-homologous links in Seifert fibered spaces may be represented combinatorially via labeled diagrams. We introduce an additional condition on a labeled link diagram and prove that it is equivalent to the existence of a rational Seifert surface for the link. In the case when this condition is satisfied, we generalize Seifert's algorithm to explicitly construct a rational Seifert surface for any rationally null-homologous link. As an application of the techniques developed in the paper, we derive closed formulae for the rational Thurston-Bennequin and rotation numbers of a rationally null-homologous Legendrian knot in a contact Seifert fibered space.
\end{abstract}

\section{Introduction}

This paper studies rationally null-homologous links in Seifert fibered spaces, with the goal of extending techniques from classical knot theory to a more general setting. Previous work in this vein includes Gilmer's [1993] signatures for rationally null-homologous links and Calegari and Gordon's [2009] classification of knots with small rational genus. More generally, recent work on the Berge conjecture has shown that the study of rationally null-homologous links is important for understanding Dehn surgery questions; see, for example, [Hedden 2011; Baker et al. 2008]. Rationally null-homologous knots are also interesting in a contact geometric setting. For example, Baker and Etnyre [2012] generalized the definition of classical invariants for Legendrian knots to the case of rational homology three-spheres and classified rational Legendrian unknots, and Cornwell [2012] has studied Bennequin-type inequalities in lens spaces. Our interest in this topic was also prompted by contact geometry [Licata and Sabloff 2010], but we hope the

Licata is partially supported by NSF grant DMS-1237324. Sabloff is partially supported by NSF grant DMS-0909273. This material is based upon work supported by the NSF under agreement No. DMS-0635607. Any opinions, findings, and conclusions or recommendations expressed in this material are those of the authors and do not necessarily reflect the views of the National Science Foundation.

MSC2010: primary 57M27; secondary 57R17.

Keywords: Seifert surface, Seifert fibered space, Legendrian knot. 
techniques developed in this paper will find applications within the wider context of link theory in rational homology three-spheres.

Just as a link in $\mathbb{R}^{3}$ is often studied via the combinatorics of its planar projection, we consider the projection of a link in a Seifert fibered space to its two-dimensional orbifold base. As we show in Section 2A, labeling this projection with some ancillary data permits the topological type of the link to be recovered. Turaev initiated this "shadow" approach in the case of links in an $S^{1}$ bundle over a surface, and the extension to $S^{1}$ bundles over orbifolds answers a question of his [Turaev 1992].

After discussing labeled link diagrams, we will introduce two further combinatorial objects: a formal rational Seifert surface is an assignment of an integer to each complementary component of the labeled link projection, while a compatible fiber distribution is an assignment of integers to each quadrant around each double point of a labeled diagram. The precise definitions are given in Section 3 and allow us to state the following theorems:

Theorem 1.1. If $L$ is rationally null-homologous in a Seifert fibered space, then any labeled diagram for $L$ admits a formal rational Seifert surface with a compatible fiber distribution.

Theorem 1.2. If a labeled diagram for $L$ admits a formal rational Seifert surface with a compatible fiber distribution, then $L$ bounds a rational Seifert surface in $M$.

It is clear that $L$ bounding a rational Seifert surface implies that $L$ is rationally null-homologous; thus, these two theorems also show that the existence of a formal rational Seifert surface with a compatible fiber distribution is equivalent to the geometric condition that $L$ is rationally null-homologous.

A key construction in this paper is a generalization of Seifert's algorithm for links in $\mathbb{R}^{3}$ to rationally null-homologous links in Seifert fibered spaces. This algorithm, which provides the proof of Theorem 1.2, explicitly constructs a rational Seifert surface in $M$ from the given combinatorial data. The algorithm is described in Section 4.

In the final section, we turn our attention to the special case of a Legendrian knot in a Seifert fibered space equipped with a transverse, $S^{1}$-invariant contact structure. (This setting was studied in more detail in [Licata and Sabloff 2010].) As an application of the algorithm defined in Section 4, we compute the rational classical invariants of a Legendrian knot from its labeled diagram; this result generalizes the familiar formulae for classical invariants in the standard contact $\mathbb{R}^{3}$.

Proposition 1.3. Let $K$ be a rationally null-homologous Legendrian knot in a contact Seifert fibered space. The rational rotation number of $K$ may be computed directly from a formal rational Seifert surface, and the rational Thurston-Bennequin number of $K$ may be computed directly from a compatible fiber distribution.

See Proposition 5.1 for a more precise statement. 


\section{Labeled diagrams}

2A. Background. We view Seifert fibered spaces as $S^{1}$ bundles over two-dimensional orbifolds, following the notational conventions of [Lisca and Matić 2004; Massot 2008].

Let $\Sigma^{\prime}$ be an orientable surface of genus $g$, possibly with boundary, with $r+1$ discs removed from its interior. Orient the new components of $\partial \Sigma^{\prime}$ as the boundary of the missing disc, and let $M^{\prime}=\Sigma^{\prime} \times S^{1}$. The first homology groups of the boundary tori of $M^{\prime}$ are generated by classes $\left\langle m_{i}, \ell_{i}\right\rangle$, with $\bigcup_{i} m_{i}=\left[\partial \Sigma^{\prime} \times\{\mathrm{pt}\}\right]$ and $\ell_{i}=\left[\{\mathrm{pt}\} \times S^{1}\right]$, oriented so that $m_{i} \cdot \ell_{i}=1$. This orients all the fibers in $M$.

For $1 \leq i \leq r$, let $\alpha_{i}$ and $\beta_{i}$ be relatively prime integers satisfying $0<\beta_{i}<\alpha_{i}$. Glue a solid torus $W_{i}$ to the $i$-th boundary component of $M^{\prime}$ so that the image of a meridian represents the homology class $\alpha_{i} m_{i}+\beta_{i} \ell_{i}$. To the remaining boundary component, glue a solid torus so that the meridian is sent to a curve representing the class of $m_{0}+b \ell_{0}$. The fiber structure on the boundary of $M^{\prime}$ extends uniquely to a fiber structure on the interior of the surgery solid tori, and the resulting identification space $M$ is said to have Seifert invariants $\left(g, b ;\left(\alpha_{1}, \beta_{1}\right), \ldots,\left(\alpha_{r}, \beta_{r}\right)\right)$. Note if $\Sigma^{\prime}$ has boundary, then $M$ has an $S^{1}$-fibered boundary.

Every orientable Seifert fibered space with an orientable fiber space can be realized via this construction; given two Seifert invariants, it is straightforward to determine whether they correspond to the same Seifert fibered manifold [Orlik 1972]. The rational Euler number of a Seifert fibered space with Seifert invariants $\left(g, b ;\left(\alpha_{1}, \beta_{1}\right), \ldots,\left(\alpha_{r}, \beta_{r}\right)\right)$ is the rational number

$$
e(M)=-b-\sum_{i=1}^{r} \frac{\beta_{i}}{\alpha_{i}} .
$$

2B. Labeled diagrams. Let $L$ be an oriented link in a Seifert fibered space $M$ with Seifert invariants $\left(g, b ;\left(\alpha_{1}, \beta_{1}\right), \ldots,\left(\alpha_{r}, \beta_{r}\right)\right)$. We suppose throughout that $L$ is generic (everywhere transverse to the fibers and with transverse crossings), and we let $\left(\Sigma, \Gamma_{L}\right)$ denote the image of $(M, L)$ under the quotient map $\pi$ sending each fiber to a point. To recover the isotopy class of $L$ from this projection, we will use a labeled diagram; this notion was introduced in [Sabloff 2003] and is similar to Turaev's notion of a shadow for a link in a circle bundle [Turaev 1992].

The fiber over a double point of $\Gamma_{L}$ is separated by its intersections with $L$ into two oriented chords, and we systematically select a preferred chord at each crossing. Near a crossing, there is a unique quadrant which is coherently and positively oriented by $L$. Declare this quadrant and the opposite quadrant to be positive, and declare the adjacent quadrants to be negative. When the oriented boundary of a positive (respectively, negative) quadrant is lifted to segments of $L$ connected by a chord, the preferred chord is the one traversed positively (negatively). 
Given a region $R$ in $\Sigma \backslash \Gamma_{L}$, let $A_{R}$ be the least common multiple of the orders of the orbifold points (i.e., the $\alpha_{i}$ ) in $R$; if $R$ contains no orbifold points, set $A_{R}=1$. Let $S_{R}$ be a compact surface with boundary, and let $\sigma_{R}: S_{R} \rightarrow \Sigma$ be an embedding on its interior onto $R$. Consider the orbibundle $\sigma_{R}^{*} M$ over $S_{R}$. The subcurves of the image of $L$ in $\sigma_{R}^{*} M$ which project to $\partial S_{R}$ may be concatenated with the preferred chords over the corners to yield a closed curve $L_{R}$ in $\partial \sigma_{R}^{*} M$. Orient $L_{R}$ so that the orientation induced on its projection to $S_{R}$ agrees with that of $\partial S_{R}$. Let $\widetilde{S}_{R}$ be the $A_{R}$-fold branched covering $S_{R}$. Use the covering map to pull the bundle $\sigma_{R}^{*} M$ back to $\widetilde{S}_{R}$. This lifts $L_{R}$ to a closed 1-manifold $\widetilde{L}_{R}$ in an honest $S^{1}$ bundle over $\widetilde{S}_{R}$. From now on, we will deliberately conflate $S_{R}$ with $R$ itself and refer to the pullback bundles as $M_{R}$ over $R$ and $\widetilde{M}_{R}$ over $\widetilde{R}$.

Let $\gamma_{1} \times \cdots \times \gamma_{k_{R}}: S^{1} \sqcup \cdots \sqcup S^{1} \rightarrow S^{1} \times \partial \widetilde{R}$ denote the map whose image is $\widetilde{L}_{R}$. Choose a trivialization of $S^{1} \times \widetilde{R}$ and let $\iota: S^{1} \times \partial \widetilde{R} \hookrightarrow S^{1} \times \widetilde{R}$ denote the inclusion. Finally, let $p: S^{1} \times \widetilde{R} \rightarrow S^{1}$ be projection to the first factor.

Definition 2.1. Given a region $R$, the defect $n(R)$ of the region is

$$
n(R)=\frac{1}{A_{R}} \sum_{i=1}^{k_{R}} \operatorname{deg}\left(p \circ \iota \circ \gamma_{i}\right) .
$$

It is immediate from the definition that $n(R)=0$ if and only if the (multi)curve $\widetilde{L}_{R}$ bounds a section of the $S^{1}$ bundle. In fact, this implies that the defect is independent of the chosen trivialization.

It follows from this definition that the defect is additive on regions. When $R$ contains no orbifold points, then the defect $n(R)$ is an integer; in general, the defect contains information about the Euler number of $M_{R}$.

Lemma 2.2. The difference between the defect $n(R)$ and the Euler number $e\left(M_{R}\right)$ is an integer: $n(R)-e\left(M_{R}\right) \in \mathbb{Z}$.

Proof. Recall that each exceptional fiber $F^{\prime}$ can be viewed as the core of a solid torus where Dehn surgery was performed on some regular fiber $F$. Let $K$ be a loop bounding a meridional disc in a regular neighborhood of $F$. After performing $(\alpha, \beta)$ surgery, $K$ intersects a meridian of the surgered torus $-\beta$ times, so the defect of the region bounded by $K$ is $-\beta / \alpha$.

Now let $K_{1}, \ldots, K_{l}$ be small loops in $R$ around the $l$ exceptional fibers in $M_{R}$. The multicurve $\partial R \cup\left(\bigcup K_{i}\right)$ bounds a region with no orbifold points, and hence has an integral defect $d$. Since the defect is additive, we see that

$$
n(R)=d-\sum_{i=1}^{l} \frac{\beta_{i}}{\alpha_{i}}=d^{\prime}+e\left(M_{R}\right)
$$

for some $d^{\prime} \in \mathbb{Z}$. 

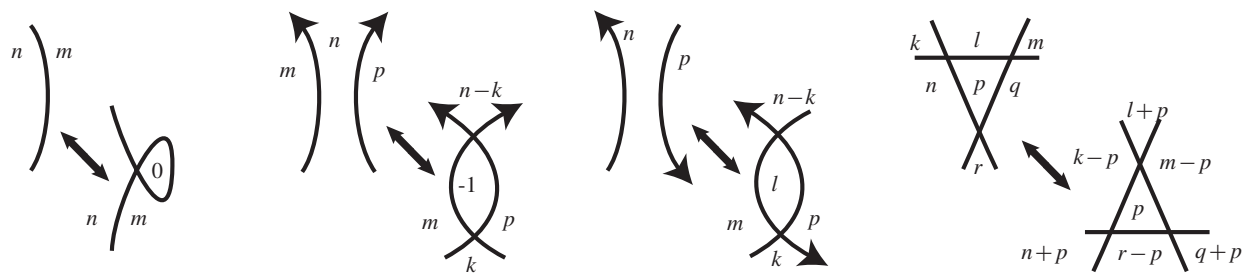

Figure 1. Labeled Reidemeister moves.

We say that a diagram $\left(\Sigma, \Gamma_{L}\right)$ is labeled when it is decorated with a defect in each region and with the fiber invariants associated to each orbifold point. Abusing notation, we will refer to both the projection and the labeled diagram by $\Gamma_{L}$. Isotopy of the link changes the labeled diagram in one of several ways. Figure 1 shows labeled Reidemeister moves for links in a Seifert fibered space; these correspond to isotopies of $L$ in the complement of the exceptional fibers. When a strand of $L$ passes through an exceptional fiber of type $(\alpha, \beta)$ the labeled diagram changes by a teardrop move which wraps $\Gamma$ around the orbifold point $\alpha$ times. See Figure 2.

In order to label the new regions created by a teardrop, we assume that the isotopy occurs in an arbitrarily small neighborhood of the exceptional fiber. The defect is therefore completely determined by the preferred chords at the new crossings. We may choose a local metric on the solid torus over the neighborhood of an orbifold point so that each regular fiber has length 1 and the exceptional fiber has length $1 / \alpha$. With such a choice, the chords created by the teardrop have lengths in the set $\{1 / \alpha, 2 / \alpha, \ldots(\alpha-1) / \alpha\}$.

The defect of a region is the signed sum of the lengths of the chords assigned to its corners, where the sign is positive at coherent corners and negative otherwise. Since the innermost region of the teardrop has a coherent corner, it follows from
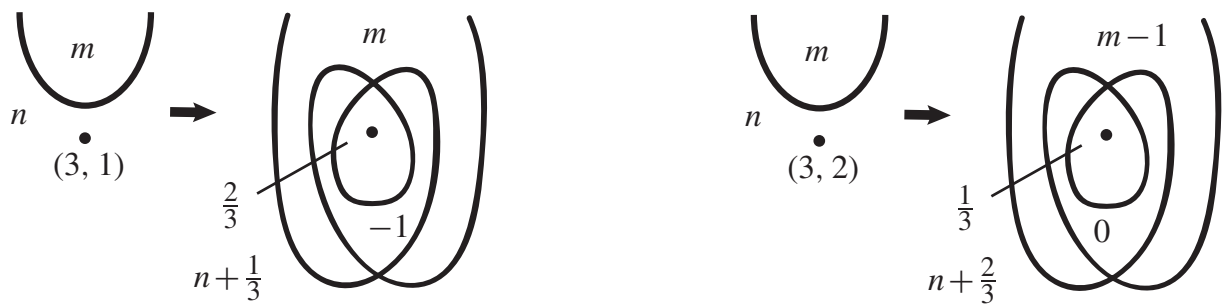

Figure 2. Labeled teardrop moves for $\alpha=3$. Left: Since the defect of the innermost region is $k / 3$ with $k \equiv-1$ modulo 3 , the length of the innermost chord is $\frac{2}{3}$. The defect of the nextinnermost region is an integer, so the length of the other preferred chord satisfies $-2-2+j \equiv 0$ modulo 3. Right: The inner chord has length $\frac{1}{3}$ and the outer chord has length $\frac{2}{3}$. 
Lemma 2.2 that the defect of this region is $(\alpha-\beta) / \alpha$. The defects of the other regions are determined by the signs of the corners and the requirement that the defect of any region not containing an orbifold point is integral.

We say that two labeled diagrams are equivalent if they differ only by sequence of surface isotopies in $\Sigma$, labeled Reidemeister moves, or labeled teardrop moves. The discussion above, together with the classical Reidemeister theorem, establishes the following lemma:

Lemma 2.3. If two generic links in $M$ are isotopic, then their labeled diagrams are equivalent.

Remark 2.4. Although it is possible to define an inverse for the teardrop move, we present it as unidirectional; passing the innermost strand of the teardrop back across the fiber introduces a second teardrop, and a sequence of Reidemeister II moves returns a projection isotopic to the original one. See Figure 3 for an example.

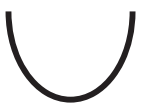

-
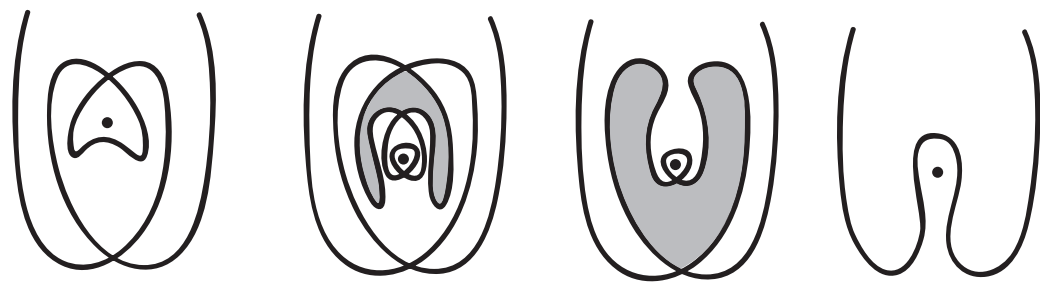

Figure 3. Composing two teardrop moves with Reidemeister II moves (through the shaded regions) returns a diagram isotopic to the original.

Next, we define a diagram move that preserves $\Gamma$ but alters the defects of a pair of adjacent regions. Following Turaev, we say fiber fusion is the operation that replaces an oriented segment of $L$ with a segment that has the same projection but travels once around the fiber.

We define an action of $H_{1}(\Sigma)$ on the set of oriented generic links $\mathscr{L}(M)$ as follows: let $\gamma$ be a generic simple closed curve on $\Sigma$ that represents a class $[\gamma] \in$ $H_{1}(\Sigma)$; in particular, we assume that $\gamma$ intersects $\pi(L)$ transversely in finitely many points and misses the double points of $\pi(L)$ and the orbifold points of $\Sigma$. Construct the link $\gamma \cdot L$ by performing fiber fusion on $L$ in a neighborhood of each point of $\gamma \cap \pi(L)$, where the sign of intersection dictates the sign of the fusion.

Lemma 2.5. The isotopy type of the link $\gamma \cdot L$ depends only on the class $[\gamma] \in H_{1}(\Sigma)$. Proof. The proof is the same as that in [Turaev 1992].

We note that the labeled diagrams associated to $L$ and to $\gamma \cdot L$ have the same defects; this follows from the fact that for each region $R$, the closed loop $\gamma$ intersects $\partial R$ zero times algebraically. Consequently, a labeled diagram of genus 
greater than zero cannot determine an isotopy class of link. We show next that each labeled diagram corresponds to an equivalence class of links related by this $H_{1}(\Sigma)$ action.

Let $\bar{\alpha}=\left(\alpha_{1}, \alpha_{2}, \ldots \alpha_{k}\right)$ be a list of the orders of the orbifold points on $\Sigma$. Pick a list $\bar{\beta}=\left(\beta_{1}, \beta_{2}, \ldots \beta_{k}\right)$ such that $\left(\alpha_{i}, \beta_{i}\right)$ are relatively prime and $1 \leq \beta_{i}<\alpha_{i}$. Let $\mathscr{D}(\Sigma, q, \bar{\alpha}, \bar{\beta})$ denote the set of labeled diagrams whose defects sum to $q$ and satisfy Lemma 2.2 in each region.

Theorem 2.6. Let $M$ be a Seifert fibered space with exceptional fiber invariants $\left\{\left(\alpha_{i}, \beta_{i}\right)\right\}$. There is a bijective correspondence between the set $\mathscr{D}(\Sigma, e(M), \bar{\alpha}, \bar{\beta})$, up to equivalence, and the set $\mathscr{L}(M)$, up to isotopy and the action of $H_{1}(\Sigma)$.

In the absence of exceptional fibers, we note that this result follows from a theorem of Turaev which establishes a bijection between his "shadow links" and isotopy classes of links in $M$, up to the action of $H_{1}(\Sigma)$. To see the theorem in this special case, we describe a bijection between labeled diagrams and shadow links. Let $R$ be a region of $\Sigma \backslash \Gamma$ with $p(R)$ positive corners and $q(R)$ negative corners with respect to the preferred chords. In the notation of [Turaev 1992], $\alpha=2 n(R)-p(R)+q(R)$ and $\beta=p(R)+q(R)$. It follows that the "gleam" of $R$ is $p(R)-n(R)$.

Proof of Theorem 2.6. As a first step, we show for a given labeled diagram in $\mathscr{D}(\Sigma, e(M), \bar{\alpha}, \bar{\beta})$, one may always find a link $L$ realizing this diagram.

Fix a labeled diagram $(\Sigma, \Gamma) \in \mathscr{D}(\Sigma, e(M), \bar{\alpha}, \bar{\beta})$. One may easily find a link $L$ in $M$ which projects to $\Gamma$, and by Lemma 2.2, the defect of any region will differ from the Euler number of the bundle over that region by an integer. We induct on the number of crossings to show that $L$ may be modified so that its defect in each region agrees with the given label. For the base case, consider a diagram consisting of a collection of disjoint embedded circles. Selecting an arbitrary component of $\Sigma \backslash \Gamma$ to be "outermost" defines a partial order on the components of $\Gamma$ by counting the minimal intersection number with $\Gamma$ of an arc connecting a point on the chosen component to a point in the outermost region. Perform fiber fusions on the curves of $L$ which project to the boundary of any innermost region in order to adjust its defect to the given label. Proceed outward, region by region. Upon reaching the outermost region, there will be no free edges available for fiber fusion, but since each fusion operation preserves the sum of the labels, the defect of the outermost region will automatically agree with the given label.

Now suppose that for any labeled diagram with fewer than $n$ crossings, we can find a link $L \subset M$ whose defects agree with the labels. Let $(\Sigma, \Gamma) \in \mathscr{D}(\Sigma, e(M), \bar{\alpha}, \bar{\beta})$ have $n$ crossings. Resolve one crossing so as to preserve the orientation of $\Gamma$ and apply the inductive hypothesis to construct a link $L^{\prime}$ whose defects agree with the labels. Replacing the crossing either preserves the number of regions, in which 


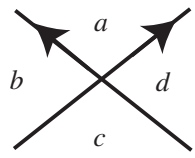

1

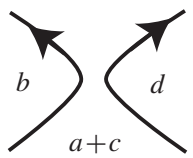

2

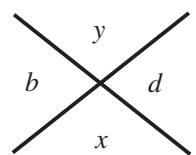

3

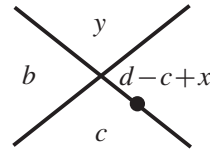

4

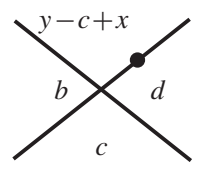

5

Figure 4. Given a labeled diagram (1), resolve a crossing of $(\Sigma, \Gamma)$ in order to apply the inductive hypothesis (2). Replace the crossing (3), noting that $x+y=a+c$. Finally, perform fiber fusions to $L^{\prime}$ until its defects are as desired $(4,5)$.

case we are done, or splits one region into two pieces. In the latter case, Figure 4 indicates how to perform fiber fusions to construct the desired $L$.

As in [Turaev 1992], the remainder of the proof of Theorem 2.6 follows from two further steps. The first step shows that any two isotopy classes of links which correspond to the same labeled diagram are related by the action of $H_{1}(\Sigma)$. The second step establishes that two generic links corresponding to equivalent labeled diagrams are related by a sequence of fiber fusions and isotopies. Turaev's arguments apply with little modification to both cases; in the second case, we additionally note that any teardrop move on labeled diagrams can be realized by a local isotopy of the link across an exceptional fiber.

\section{Combinatorics for rational Seifert Surfaces}

In this section, we develop a combinatorial description of a rational Seifert surface for a rationally null-homologous link $L$. The description has the form of two decorations of the labeled diagram $\Gamma_{L}$ of $L$ : a "formal rational Seifert surface" and a compatible "fiber distribution". The two decorations will be used in the next section to describe a generalization of the Seifert algorithm.

3A. Two decorations of labeled diagrams. A surface in a Seifert fibered space is said to be horizontal if it is everywhere transverse to the fibers; we relax this condition slightly and consider rational Seifert surfaces which are transverse except near fibers over double points of $\Gamma$. The idea of the first decoration is that any such surface assigns a multiplicity to each region $R$. Conversely, we may characterize the sets of multiplicities on $\Sigma$ which are induced by such a surface using the following combinatorial object:

Definition 3.1. A formal rational Seifert surface $\boldsymbol{m}$ of order $r$ is an assignment of an integral multiplicity $m\left(R_{j}\right)$ to each region $R_{j}$ of $\Sigma \backslash \Gamma$ that satisfies the following conditions.

(1) The least common multiple $A_{R_{j}}$ of the orders of the orbifold points in $R_{j}$ divides $m\left(R_{j}\right)$. 
(2) If $R_{k}$ and $R_{l}$ share an edge oriented as $\partial R_{k}$, then

$$
m\left(R_{k}\right)-m\left(R_{l}\right)=r .
$$

(3) Summing over all regions,

$$
\sum_{j} m\left(R_{j}\right) n\left(R_{j}\right)=0 .
$$

A formal rational Seifert surface may be viewed as a secondary labeling on a link diagram, and we introduce a tertiary labeling as well. Let $x_{j}^{i}$ denote a corner of the region $R_{j}$ at the $i$-th crossing. (It is possible for a single region to fill more than one corner at a given crossing, but for notational convenience, we avoid introducing a third index to distinguish them.)

Definition 3.2. Given a formal rational Seifert surface $\boldsymbol{m}$ for a labeled diagram $\Gamma$, a fiber distribution compatible with $\boldsymbol{m}$ is an assignment $\boldsymbol{f}$ of integers $f\left(x_{j}^{i}\right)$ to the corners of regions of $\Sigma \backslash \Gamma$ which satisfies the following properties:

(1) For each region $R_{j}$ with corners $x_{j}^{i}$ for $i \in \mathscr{C}_{R}=\left\{i_{1}, \ldots, i_{k_{R}}\right\}$,

$$
m\left(R_{j}\right) n\left(R_{j}\right)+\sum_{i \in \mathscr{C}_{R}} f\left(x_{j}^{i}\right)=0 .
$$

(2) For each crossing labeled $i$ with incident regions $R_{j_{1}}, \ldots, R_{j_{4}}$,

$$
\sum_{k=1}^{4} f\left(x_{j_{k}}^{i}\right)=0 .
$$

Rational formal Seifert surfaces and their fiber distributions are best understood in terms of a special cell decomposition of $M$, which is constructed in Section 3B. As motivation, however, one may view the rational formal Seifert surface as describing how a surface interacts with the base orbifold $\Sigma$, whereas a fiber distribution captures its interaction with the bundle structure of $M$.

Example 3.3. The figure shows a labeled diagram for a knot in $L(5,2)$, together with a rational formal Seifert surface and fiber distribution.

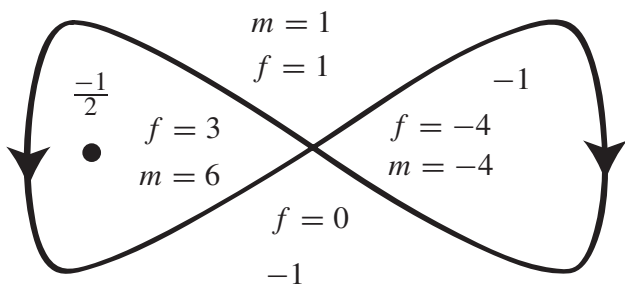

Figure 5. A labeled diagram for $K \subset L(5,2)$, together with a formal rational Seifert surface and a compatible fiber distribution. 
We will use this example to illustrate the generalized Seifert algorithm in Section 4.

3B. A cell decomposition for $M$. In this section, we construct a cell decomposition of $M$ using data from the link $L$. We begin by enlarging the graph $\Gamma_{L}$ so that each complementary region is homeomorphic to a disc and contains at most one orbifold point. If a region has nontrivial topology or contains more than one orbifold point, subdivide it using a collection of $\operatorname{arcs} \Gamma_{0} \subset \Sigma$ whose endpoints lie on $\Gamma_{L}$; let $\bar{\Gamma}$ denote the graph $\Gamma_{L} \cup \Gamma_{0}$. Lift the arcs of $\Gamma_{0}$ to arcs $L_{0}$ in $M$ whose endpoints lie on $L$. The link $L$, the arcs $L_{0}$, and the fibers over each vertex of $\bar{\Gamma}$ form a 1-complex in $M$.

The 2-cells of $M$ are of two types. First, for each edge $e$ of $\bar{\Gamma}$, let $D_{e}$ be the preimage of $e$ in $M$, thought of as a disc whose boundary consists of the fibers over the ends of the edge, together with two oppositely oriented copies of the corresponding segment of $L \cup L_{0}$. Refer to this type of cell as vertical. Second, for each region $R$ of $\Sigma \backslash \bar{\Gamma}$, we construct the regional cell $D_{R}$ as follows. Denote the fibers over double points in $\partial R$ by $\left\{F_{i}\right\}$. The lifted curve $L_{R}$ satisfies $\left[A_{R} L_{R}-\sum b_{i} F_{i}\right]=0 \in H_{1}(M)$ for any $b_{i}$ such that $\sum b_{i}=A_{R} n(R)$. The 1-chain $A_{R} L_{R}-\sum b_{i} F_{i}$ bounds a disc in $M_{R}$, and we include this as the 2-cell $D_{R}$.

The remainder of $M$ consists of 3-balls that come from removing a meridian disc from the solid torus over each region of $\bar{\Gamma}$.

\section{C. Proof of Theorem 1.1. Recall the statement of Theorem 1.1:}

If $L$ is rationally null-homologous in a Seifert fibered space, then any labeled diagram for L admits a formal rational Seifert surface with a compatible fiber distribution.

Proof. Suppose that $L$ is rationally null-homologous with order $r$. The link $L$ has an obvious representative (which we shall also call $L$ ) as a 1-chain in the cell decomposition described above. Hence, there exists a 2-chain $S$ such that $\partial S=r L$. For each region $R_{j} \in \Sigma \backslash \bar{\Gamma}$, let $c_{j}$ denote the coefficient of $D_{j}$ in $S$. Assign the multiplicity $m\left(R_{j}\right)$ to be $c_{j} A_{R_{j}}$.

We begin by verifying condition (1) of Definition 3.1. It is clearly satisfied on disc components of $\Sigma \backslash \bar{\Gamma}$. Now suppose that $R_{1}$ and $R_{2}$ in $\Sigma \backslash \bar{\Gamma}$ are separated by the edge $e_{0} \in \Gamma_{0}$. The assumption that $\partial S=r L$ implies that this edge has multiplicity 0 in $\partial S$, so $m\left(R_{1}\right)=m\left(R_{2}\right)$. This shows that the multiplicities are well-defined on components of $\Sigma \backslash \Gamma_{L}$. Since $\alpha_{j} \mid m\left(R_{j}\right)$ for $j=1,2$ and $m\left(R_{1}\right)=m\left(R_{2}\right)$, condition (1) is satisfied on $R_{1} \cup R_{2}$, and an inductive argument shows that it holds for all components of $\Sigma \backslash \Gamma_{L}$.

Adding a vertical 2-cell to a chain does not change the coefficient of any edge of $L$ in the boundary 1-chain. Each edge of $L$ appears $r$ times in $\partial S$, so the difference in multiplicities between the two adjoining regional cells is $r$, establishing condition (2). 
Finally, we show that condition (3) of Definition 3.1 holds. By construction, the boundary of each regional cell consists of $A_{R_{j}} L_{R_{j}}$ and $-A_{R_{j}} n\left(R_{j}\right)$ copies of the fiber. Thus the total number of copies of the fiber coming from regional 2cells is $\sum_{j}-m\left(R_{j}\right) n\left(R_{j}\right)$. The addition of any vertical 2-cell preserves this sum, and the assumption that $\partial S=r L$ implies that the copies of the fiber must cancel algebraically: $\sum_{j} m\left(R_{j}\right) n\left(R_{j}\right)=0$.

To construct a compatible fiber distribution $f$, consider a quadrant $x_{j}^{i}$ of a crossing $i$ lying in the region $R_{j}$. Suppose that this quadrant lies to the right of the oriented edges $\mathscr{E}\left(x_{j}^{i}\right)$ of $\bar{\Gamma}$; note that this set may be empty and has at most two elements. We then define $f\left(x_{j}^{i}\right)$ to be

$$
f\left(x_{j}^{i}\right)=-c_{j} b_{i}+\sum_{e \in \mathscr{E}\left(x_{j}^{i}\right)} \epsilon_{e} f_{e},
$$

where the integer $b_{i}$ comes from the construction of the regional cell $D_{R_{j}}, f_{e}$ is the coefficient of the vertical cell $D_{e}$ in $S$, and $\epsilon_{e}= \pm 1$ is positive if and only if the head of $e$ is incident to the double point $i$.

Condition (1) of Definition 3.2 now follows from two facts. First, observe that $c_{j} \sum b_{i}=c_{j} A_{R_{j}} n\left(R_{j}\right)=m\left(R_{j}\right) n\left(R_{j}\right)$. Second, note that each edge with $R_{j}$ on its right contributes $f_{e}$ to the sum associated to the quadrant at its head and $-f_{e}$ to the sum associated to the quadrant at its foot; thus, the contributions coming from the vertical 2-cells cancel around any given region. Condition (2) holds because $\sum_{k=1}^{4} f\left(x_{j_{k}}^{i}\right)$ is the coefficient of the fiber over the double point $i$ in $\partial S$, but we know that $\partial S=r L$, and hence this coefficient must vanish.

Remark 3.4. One may show that every formal rational Seifert surface admits a compatible fiber distribution, a fact which permits a stronger formulation of Theorem 1.2. The proof is by induction on the number of double points of $\Gamma$, and we leave the details to the reader.

\section{Seifert algorithm for links in $S^{1}$ orbifold bundles}

Given a formal rational Seifert surface $\boldsymbol{m}$ and a fiber distribution $\boldsymbol{f}$ for a rationally null-homologous link of order $r$, we construct a rational Seifert surface of the same order. The classical Seifert algorithm for links in $\mathbb{R}^{3}$ proceeds in three steps: first, one resolves the crossings in a projection of the link to obtain a collection of Seifert circles in the plane. Second, one views the Seifert circles as bounding disjoint embedded disks. Finally, the Seifert disks are connected by twisted bands at the crossings. The generalized algorithm for a link in a Seifert fibered space parallels the classical algorithm. As a first step, we let $D_{i}$ denote a neighborhood of the $i$-th double point of $\Gamma$ and let $U_{i}=\pi^{-1}\left(D_{i}\right)$. We use $\boldsymbol{m}$ and $\boldsymbol{f}$ to resolve the link into circles in $M \backslash \bigcup U_{i}$ (Section 4A). Next, we view these resolved circles 
as bounding embedded surfaces in $M \backslash U_{i}$ (Section 4B). Finally, we extend these surfaces across the solid tori $U_{i}$ (Section $4 \mathrm{C}$ ). We begin by establishing notation which will be useful throughout the algorithm.

For each double point of $\Gamma$, parametrize the neighborhood $D_{i}$ as a unit disc and let $C_{t}^{i}$ denote the $S^{1}$ bundle over the circle of radius $t$. Dropping the superscript when the crossing is obvious, we split the torus $C_{1}$ into annuli denoted $A_{I}, A_{\mathrm{II}}$, $A_{\mathrm{III}}$, and $A_{\mathrm{IV}}$ according to the corresponding quadrants of $\Sigma$; see Figure 6.

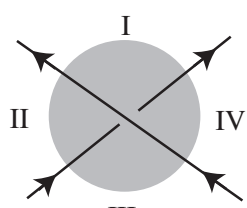

III

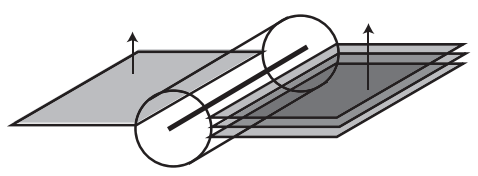

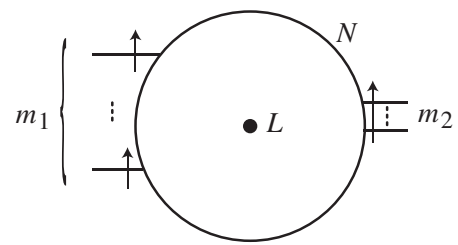

Figure 6. Left: The disc $D_{i}$ near a double point of $\Gamma$. Center: $S$ in a neighborhood of $L$ away from a double point. Right: A cross-section of $N$ between regions with multiplicities $m_{1}$ and $m_{2}$, where $m_{1}-m_{2}=r$.

Let $L_{0}$ be the arcs constructed in Section 3B. Near $L \cup L_{0}$ but away from the double points of $\bar{\Gamma}$, the local behavior of any rational Seifert surface is dictated by the multiplicities of the adjacent regions; note that the multiplicities on regions of $\Gamma$ induce multiplicities on the regions of $\bar{\Gamma}$. Let $N$ be a regular neighborhood of $L \cup L_{0}$, and suppose that the projection of a segment of $L \cup L_{0}$ separates regions with multiplicities $m_{1}$ and $m_{2}$. In this case, the rational Seifert surface $S$ intersects $\partial N m_{i}$ times on each side. Correspondingly, to each side of a cross section of $N$ we draw $m_{i}$ parallel, transversely oriented lines. The endpoints of these lines trace out $m_{i}$ parallel curve segments on $\partial N$ as the cross-section varies; see Figure 6.

4A. Resolution into Seifert circles. The first step of the construction replaces $L$ with a collection of circles. Remove the interior of $N$ and the fibered solid tori $U_{i}$ from $M$. As described above, the portions of $\partial N$ away from the $U_{i}$ and neighborhoods of the intersection points $L \cap L_{0}$ are decorated with collections of parallel curves. Near the intersection points $L \cap L_{0}$, we simply join the endpoints of corresponding parallel curves. Near the solid tori $U_{i}$, we will use $\boldsymbol{m}$ and $\boldsymbol{f}$ to construct a pattern of curves on $C_{1}^{i}$ which connect the endpoints of the parallel curves.

Fix a crossing, and for convenience, cut the corresponding solid torus along a meridional disc so that $C_{1}$ becomes a cylinder composed of four rectangles still labeled I, II, III, and IV. Orienting each rectangle as if viewed from $t>1$, decorate it with a pattern of multicurves as shown in Figure 7. Each curve is decorated with an arrow indicating its transverse orientation and by an integer weight indicating its 


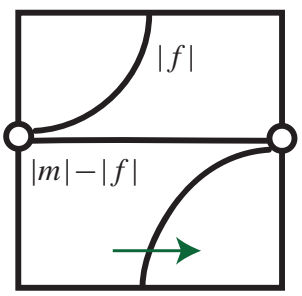

$\epsilon f<0$

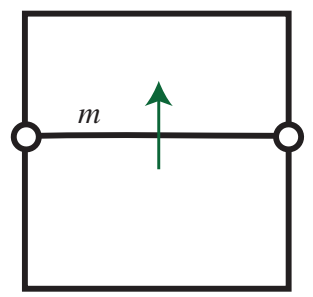

$f=0$

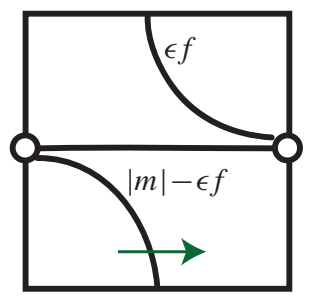

$\epsilon f>0$

Figure 7. The local models for $f>0$. The circles on the side edges are the intersections between $C_{1}$ and $N$. Changing the sign of $f$ reverses the arrows. The sign of the formal rational Seifert surface incident to $C_{1}$ is labeled by $\epsilon$.

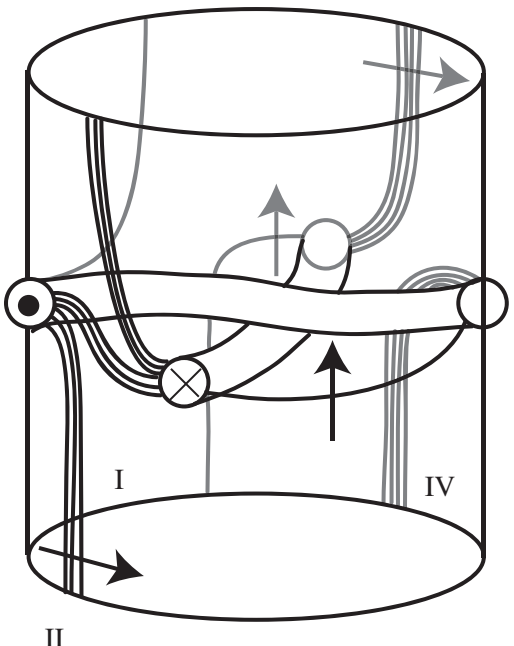

III

Figure 8. The intersection pattern on $C_{1}$ for the unique crossing in the knot from Example 3.3.

multiplicity. Reversing the arrow changes the sign of this weight. By construction, the endpoints of these curves can be glued to the endpoints of the curves on $\partial N$.

The resulting pattern of curves on $\partial\left(N \cup\left(\bigcup_{i} U_{i}\right)\right)$ will serve as our Seifert circles. Before proceeding, we note the following:

Lemma 4.1. The sum of the algebraic intersection numbers of the pattern curves with the meridian of $C_{1}$ is zero around each double point.

Proof. This follows from condition (2) of Definition 3.2. 
4B. Surfaces bounded by Seifert circles. We begin the second step by constructing surfaces in $M \backslash\left(N \cup\left(\bigcup_{i} U_{i}\right)\right)$ bounded by the Seifert circles.

Condition (1) of Definition 3.2 implies that all $m(R) / A_{R}$ Seifert circles over the boundary of a given region $R$ are null-homologous and hence bound horizontal embedded discs in $M_{R}$. By construction, the signed intersection number of each curve pattern with $\partial N \cap C_{1}$ is $r$; see Figure 6 .

To complete this step, we extend this surface over the cylinders $N \cap\left(M \backslash \bigcup U_{i}\right)$. There are two cases to consider If the multiplicities of the adjoining regions have the same sign, then we extend the embeddings of the surfaces as in Figure 9, left, for an appropriate choice of $k, l \geq 0$. In particular, if the regions in question are separated by an edge of $\pi\left(L_{0}\right)$, then the multiplicities of the adjacent regions are the same and we use $k+l=m_{-}$in the figure. If, on the other hand, the multiplicities of the adjoining regions have opposite signs, then the extension is as in Figure 9, right; in this case, there is no choice to make.
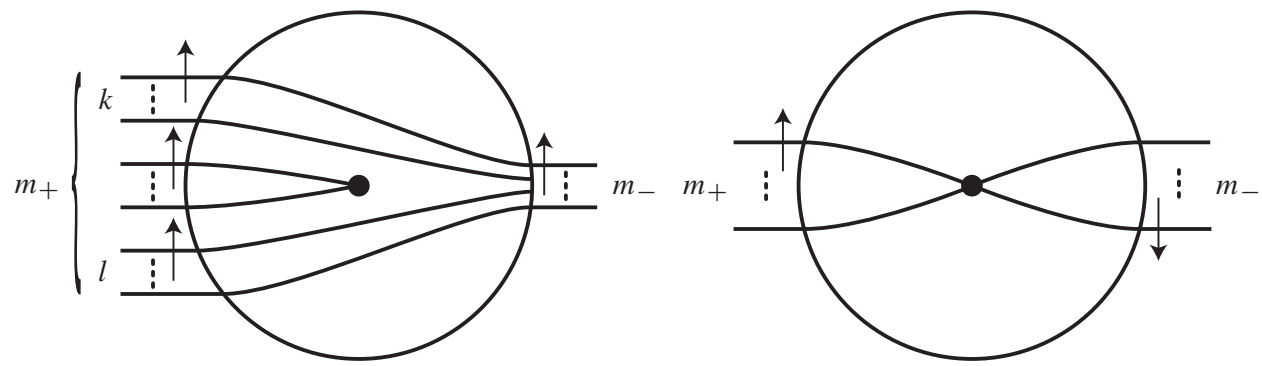

Figure 9. The extension of the rational Seifert surface across $N$ if the multiplicities of the adjoining regions have the same sign (left) and if they have opposite signs (right).

4C. Extending across solid tori over crossings. We have now constructed a surface in the complement of the crossing tori $U_{i}$. In this section, we extend the surface across each $U_{i}$ by describing how it intersects a collection of concentric cylinders $C_{t}$ of decreasing radius. Modifications to the intersection pattern describe changes in the surface. In addition to surface isotopy of the curves, we allow the following three primitive moves:

Finger moves. In the complement of $C_{t} \cap N$, we may replace a curve segment adjacent to $C_{t} \cap \partial N$ with a pair of curves ending on $C_{t} \cap \partial N$; these intersections will have opposite signs. This move preserves the topology of the surface, but pushes it locally into the neighborhood of $L$. See Figure 10.

Capping a circle. Any embedded circle may be removed from the intersection pattern. This corresponds to capping off the corresponding component of $S \cap C_{t_{0}}$ with a disc embedded in the solid torus defined by $t<t_{0}$. 

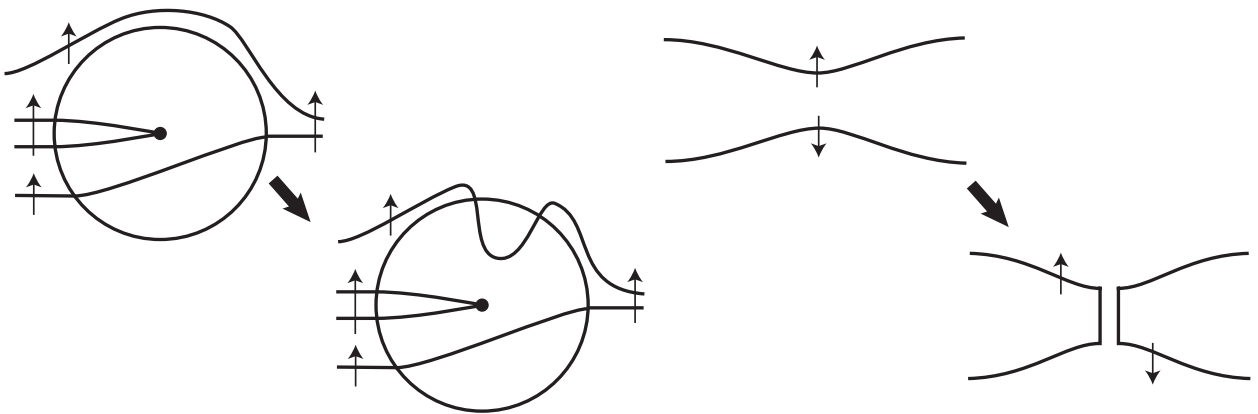

Figure 10. Left: a finger move breaks a curve in the complement of $C_{t} \cap N$ into two pieces; this corresponds to pushing the surface into $N$. Right: an oriented saddle resolution.

Saddle moves. We may perform a saddle resolution between two curves with opposite transverse orientations. This corresponds to reducing the Euler characteristic of the surface by 1 .

We will also make use of two consequences of these three moves.

Cancellation of parallel strands. Two oppositely oriented adjacent parallel strands between components of $N \cap C_{t}$ may be removed. See Figure 11.

Reconfiguration in N. Any two configurations that appear in Figure 9 are related by a sequence of saddle moves. See Figure 11 .

We now begin to extend the surface $S$ across the solid torus $U_{i}$. Isotope all the intersections of the Seifert circles with the meridian disc to the annulus $A_{\text {II }}$. Fixing these intersections, standardize the pattern of curves on $C_{t}$ via isotopy, finger moves, and cancellations of oppositely oriented parallel strands. Note that after cancellation, the configurations inside $N \cap C_{t}$ are again of the form in Figure 9.
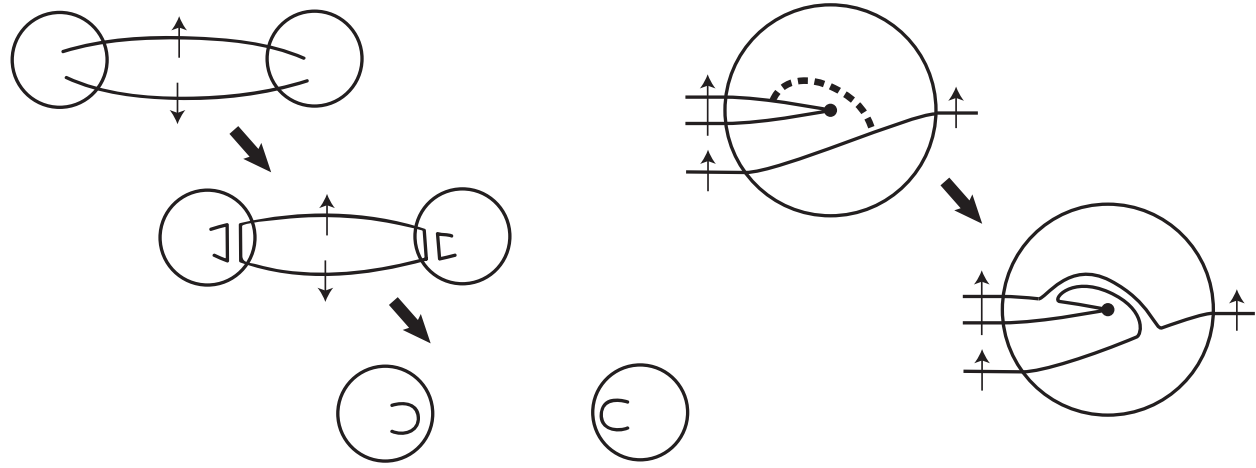

Figure 11. Left: parallel strands with opposite orientations cancel. Right: an oriented saddle move modifies the configuration inside $N$. 
Lemma 4.1 states that the algebraic intersection number of these curves with the meridian is zero, and saddle resolutions between oppositely oriented curves reduce the geometric intersection number to zero as well.

The resulting pattern may contain curves with both endpoints on the same component of $N \cap C_{t}$; these may be again be removed using sequences of the moves above, especially capping circles.

As $t \rightarrow 0$, the strands of $L$ cross; this rotates a region containing two components of $N \cap C_{t}$ by $\pi$. Further finger moves, cancellations, and isotopies yield a standard pattern consisting solely of horizontal curves. It is clear that these bound a collection of discs, completing $S$. Note that reconfigurations inside $N$ allow us to match those configurations coming from opposite sides of the intersection of one component of $N \cap U_{i}$. See Figure 12 for an example.
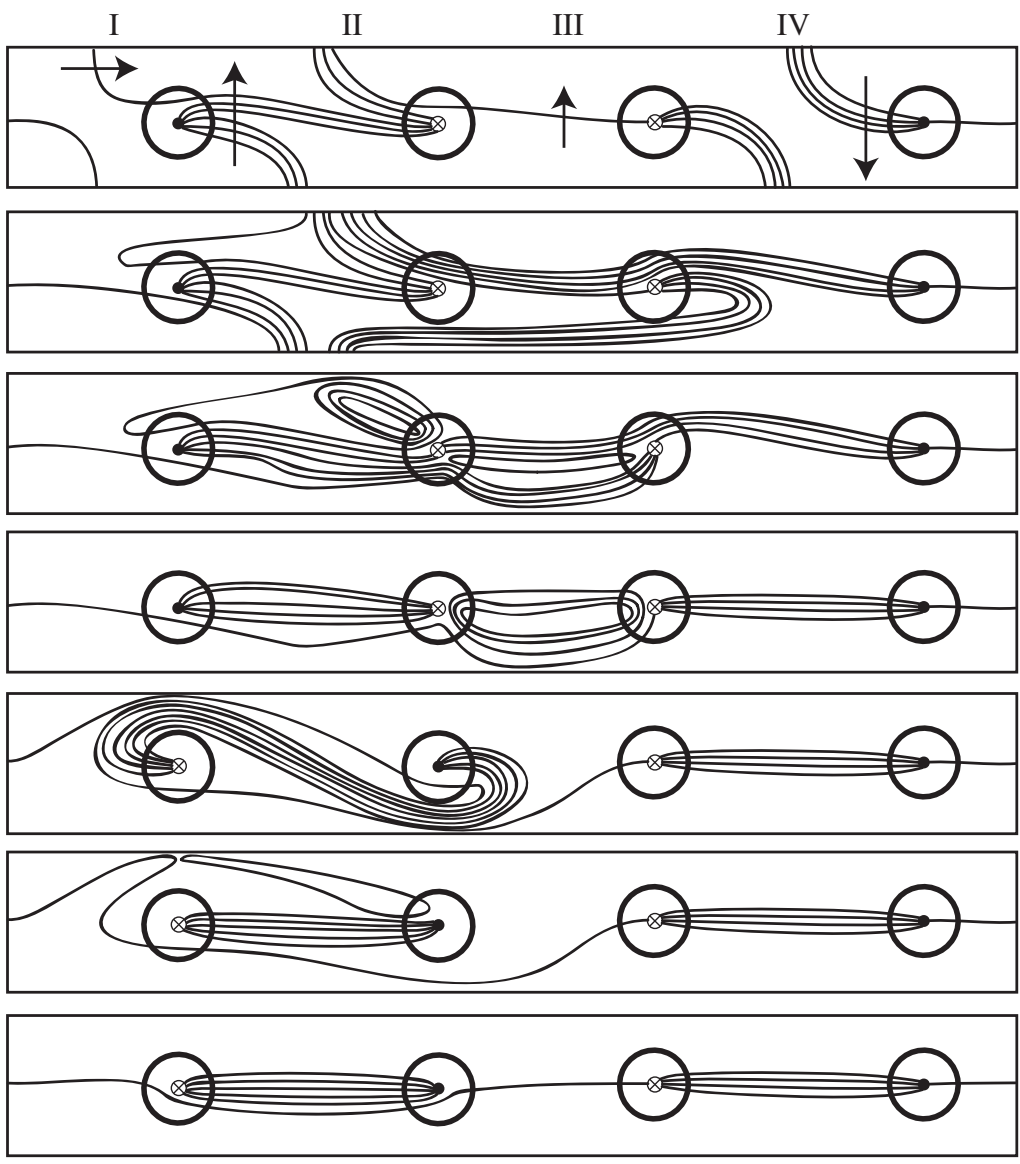

Figure 12. Continuing Example 3.3: a sequence of intersection patterns for decreasing values of $t$. 


\section{Legendrian invariants}

In this section we use the use the generalized Seifert algorithm to compute the rational classical invariants for a rationally null-homologous Legendrian knot from a formal rational Seifert surface and fiber distribution.

5A. Contact Seifert fibered spaces. We will use the phrase contact Seifert fibered space to denote an orientable Seifert fibered space over an orientable base, equipped with a contact structure $\xi$ transverse to the Seifert fibers. Such a contact structure exists whenever the rational Euler number of a Seifert fibered space is negative [Kamishima and Tsuboi 1991; Lisca and Matić 2004]. If we further specify a contact form $\alpha$ for $\xi$ with the property that its Reeb field points along the fibers (see [Licata and Sabloff 2010]), then the defect defined in Section 2B can be interpreted as an integral of the curvature form associated to $\alpha$ on the Reeb orbit space. We note that the Legendrian condition precludes the Reidemeister I move of Section 2B.

A formal rational Seifert surface $\boldsymbol{m}$ and a compatible fiber distribution $\boldsymbol{f}$ may be used to compute the rational classical invariants of a rationally null-homologous Legendrian knot in a contact Seifert fibered space. We prove this using the rational Seifert surfaces constructed in Section 4.

For each region $R_{j} \in \Sigma \backslash \Gamma$, let $\chi_{\text {orb }}\left(R_{j}\right)$ denote the orbifold Euler characteristic of $R_{j}$ as a suborbifold of $\Sigma$; recall that this quantity is defined to be

$$
\chi_{\mathrm{orb}}(R)=\chi(R)+\sum_{j=1}^{r}\left(\frac{1}{\alpha_{j}}-1\right) .
$$

Let $k_{j}$ and $l_{j}$ denote the number of double points of $\Gamma$ where $R_{j}$ fills one or three quadrants, respectively. We restate Proposition 1.3 as follows:

Proposition 5.1. The rational classical invariants of a null-homologous Legendrian knot $K$ maybe be computed from a formal rational Seifert surface $\boldsymbol{m}$ and a compatible fiber distribution $\boldsymbol{f}$ using the following formulae:

$$
\begin{aligned}
& \operatorname{rot}_{\mathbb{Q}}(K)=\frac{1}{r} \sum_{\text {regions } R_{j}} m\left(R_{j}\right)\left[\chi_{\mathrm{orb}}\left(R_{j}\right)+\frac{1}{4}\left(l_{j}-k_{j}\right)\right], \\
& \operatorname{tb}_{\mathbb{Q}}(K)=\frac{1}{r} \sum_{\text {dble pts } i}\left(-r-f_{\mathrm{II}}^{i}+f_{\mathrm{IV}}^{i}\right) .
\end{aligned}
$$

Here, $f_{*}^{i}$ denotes the label assigned by the fiber distribution to the quadrant labeled $*$ at the $i$-th crossing.

The subsequent sections discuss these invariants and develop proofs of these propositions. 
Example 5.2. The knot in Example 3.3 can be realized as a Legendrian knot whose Lagrangian projection is shown in Figure 5. To see this, begin with the unknot with maximal Thurston-Bennequin number in the standard contact $S^{3}$. Performing 1 and $\frac{1}{2}$ surgery on a pair of regular fibers yields the labeled diagram of Figure 5, and the contact form may be extended across the surgery tori so that the induced Reeb orbits are the Seifert fibers.

The results above show that this knot has rational rotation number

$$
\operatorname{rot}_{\mathbb{Q}}(K)=\frac{1}{5}\left[6\left(\frac{1}{4}\right)+1\left(\frac{1}{2}\right)-4\left(\frac{3}{4}\right)\right]=-\frac{1}{5}
$$

and rational Thurston-Bennequin number

$$
\mathrm{tb}_{\mathbb{Q}}(K)=\frac{1}{5}(-5-3-4)=-\frac{12}{5} .
$$

5B. The rational rotation number. Baker and Etnyre [2012] defined the rational rotation number of a rationally null-homologous knot by analogy with the classical rotation number for a null-homologous knot. Let $j: S \hookrightarrow M$ be a rational Seifert surface for $K$. Trivialize the pulled back contact bundle $j^{*} \xi$ over $S$ using a nonvanishing vector field $v$; since $K$ is Legendrian, $T K$ lies in the restriction of $\xi$ to $\partial S$. One may therefore define the winding number of $j^{*} T K$ :

$$
\operatorname{rot}_{\mathbb{Q}}(K)=\frac{1}{r} \operatorname{wind}_{V}\left(j^{*} T K\right) .
$$

To better understand a trivialization of $j^{*} \xi$, we will cut $S$ along its intersection with the vertical tori $\partial U_{i}$. This creates a collection of disjoint surfaces with boundary, denoted collectively by $\hat{S}$; we compute the rotation of each component individually and sum them to compute the rational rotation number of $K$. Note that cutting introduces new segments to the boundary curves; although these could be isotoped to be Legendrian, their contributions to the rotation will cancel under gluing. We may therefore ignore these segments and compute only the contributions to the rotation number of $T(\partial \hat{S})$ by $T K$.

We begin by showing that the contribution of a component $X$ of $\hat{S}$ lying in the solid torus $U_{i}$ to $\operatorname{rot}_{\mathbb{Q}}(K)$ is zero. We may assume that the complex structure on $\Sigma$ is chosen so that the arcs of $\Gamma$ intersect the boundary of the neighborhood of the double point orthogonally. Choosing the neighborhood of a fixed double point small enough, we may trivialize $T \Sigma$ over the disc $D_{i}$ with vector fields $\{v, i v\}$ so that $T K$ never coincides with the lines spanned by $v$ and $i v$. Pull back this trivialization to $\left.\xi\right|_{U_{i}}$, and then again to $\left.j^{*} \xi\right|_{X}$. With respect to this trivialization, it is obvious that $K \cap U_{i}$ contributes zero to the rotation number.

We now turn to the portions of $S$ constructed from Seifert circles in Section 4B, i.e., the components of $j(S) \cap\left(M_{R} \backslash \bigcup U_{i}\right)$. Recall that these components of $\hat{S}$ are horizontal, and hence that we may identify $T S$ and $j^{*} \xi$ on these portions. The next 
lemma extends the existing trivialization of $j^{*} \xi$ from $j(S) \cap \partial U_{i}$ and describes the contribution to $\operatorname{rot}_{\mathbb{Q}}(K)$ coming from a single region $R$.

Lemma 5.3. Suppose that the region $R$ has multiplicity $m(R)$ in a formal rational Seifert surface for $K$, and that $k$ and $l$ denote the number of double points in $\partial R$ where $R$ fills one and three quadrants, respectively. Then the contribution of $j(S) \cap\left(M_{R} \backslash \bigcup U_{i}\right)$ to $\operatorname{rot}_{\mathbb{Q}}(K)$ is $\frac{1}{r} m(R)\left[\chi_{\mathrm{orb}}(R)+\frac{1}{4}(l-k)\right]$.

Together with the discussion above, this lemma finishes the proof of the first part of Proposition 5.1.

Proof of Lemma 5.3. As a consequence of trivializing $\xi$ over the solid tori $U_{i}$, each truncated region may be replaced by the original region without affecting its contribution to the rotation.

Let $S_{R}$ be a component of $j(S) \cap M_{R}$. Note that $S_{R}$ is an $A_{R}$-fold branched cover of $R$, branched over the orbifold points of $R$. We represent a trivialization of $\left.\xi\right|_{S_{R}}$ by a nonvanishing vector field in $T S_{R}$, and we use the Poincaré-Hopf theorem to compute the winding number of $T \partial S_{R}$ with respect to this framing on the boundary. Embed $S_{R}$ as a subsurface of a closed surface $\bar{S}_{R}$ satisfying $\chi\left(\bar{S}_{R}\right)=\chi\left(S_{R}\right)+1$. Choose a vector field $v$ on $\bar{S}_{R}$ that extends the trivialization of $\xi$ in the tori $U_{i}$ and which has the property that its unique critical point $c$ lies in $\bar{S}_{R} \backslash S_{R}$. Because $S_{R}$ is a branched cover of $R$, we may use the Riemann-Hurwitz theorem to compute the Euler characteristic of $S_{R}$ :

$$
\chi\left(S_{R}\right)=A_{R}\left[\chi(R)+\sum_{i=1}^{r}\left(\frac{1}{\alpha_{i}}-1\right)\right] .
$$

The Poincaré-Hopf theorem implies that the index of $v$ at the unique critical point $c$ is

$$
\operatorname{ind}_{c} v=1+A_{R}\left[\chi(R)+\sum_{i=1}^{r}\left(\frac{1}{\alpha_{i}}-1\right)\right] .
$$

We now compute the winding number of $\partial S_{R}$ as an embedded curve with corners which encircles the singular point of the vector field (Figure 13). For simplicity, consider the curve $-\partial S_{R}$ (which bounds a neighborhood of the critical point positively). Identifying this neighborhood with a neighborhood of the origin in $\mathbb{C}$, and computing the winding number of the tangent to $-\partial S_{R}$ with respect to the translation-invariant page framing yields

$$
\operatorname{wind}_{\text {page }}\left(-\partial S_{R}\right)-A_{R}\left(\frac{k}{4}\right)+A_{R}\left(\frac{l}{4}\right)=1 .
$$

To convert the winding number with respect to the page framing to the winding number with respect to $v$, subtract the index of $c$ :

$$
\operatorname{wind}_{v}\left(-\partial S_{R}\right)=\operatorname{wind}_{\text {page }}\left(-\partial S_{R}\right)-\operatorname{ind}_{c} v .
$$



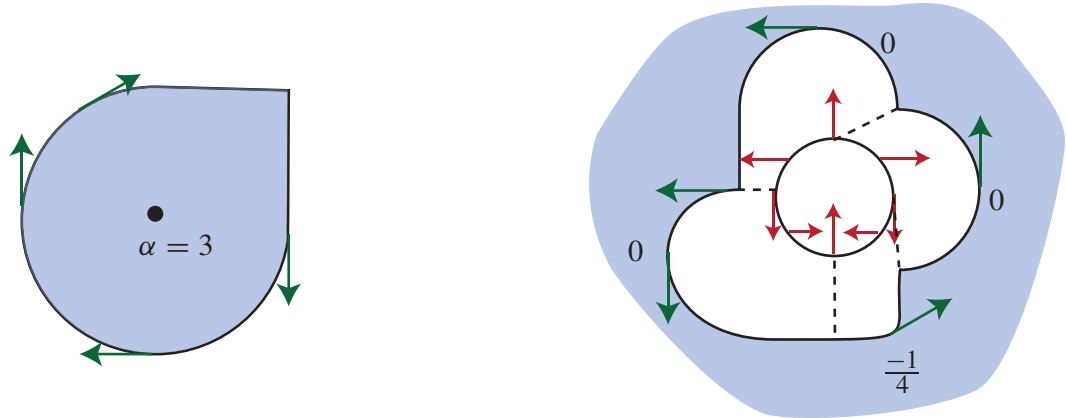

Figure 13. Left: The boundary of the shaded region is oriented as $-\partial R$, with $k=1$ and $\alpha=3$. Right: The boundary of a 3fold branched cover of $R$ embedded on a sphere. In a neighborhood of the index two critical point, $\operatorname{wind}_{\text {page }}\left(-\partial S_{R}\right)=\frac{7}{4}$ and $\operatorname{wind}_{v}\left(-\partial S_{R}\right)=\frac{-1}{4}$.

The Seifert surface is constructed locally using $m(R) / A_{R}$ copies of $S_{R}$, so the result follows from Equations (5-1), (5-4), and (5-5).

5C. The rational Thurston-Bennequin number. In this final section, we use a rational formal Seifert surface and a fiber distribution to compute the rational Thurston-Bennequin invariant. Recall from [Baker and Etnyre 2012] that the rational Thurston-Bennequin number of a Legendrian knot $K$ is defined to be the rational linking number of $K$ with a transverse push-off $K^{\prime}$ with respect to some rational Seifert surface for $K$.

Since the fibers are transverse to the contact planes, we may take $K^{\prime}$ to be the Legendrian push-off along the Reeb direction; we may think of $K^{\prime}$ as lying at the bottom of $\partial N$. Away from the double points of $\Gamma$, the conventions for how a rational Seifert surface $S$ interacts with $N$ in Figure 6 imply that there will be no intersection points. Thus, computing $\operatorname{tb}_{\mathbb{Q}}(K)$ reduces to counting intersections between $S$ and $K^{\prime}$ in the solid tori over the double points of $\Gamma$.

Proof of (5-3). As discussed above, it suffices to examine how the generalized Seifert algorithm extends the Seifert surface $S$ across a fibered neighborhood of a double point of $\Gamma$. The only interactions of $S$ and $K^{\prime}$ will be when the generalized Seifert algorithm uses finger moves to push $S$ across the bottom of $N$. The sign of these intersections may be computed combinatorially as in Figure 14. We need to count (with sign) finger moves of $S$ across the bottom of $\partial N$. It will be convenient to adopt the notation $f_{*}$ (respectively, $m_{*}$ ) to denote the value of the fiber distribution (formal rational Seifert surface) on the quadrant (region) labeled $*$.

The first step in extending $S$ requires sliding each intersection between the fiber and the top edge of $C_{t}$ into $A_{\mathrm{II}}$ and then standardizing the resulting pattern. Isotope 

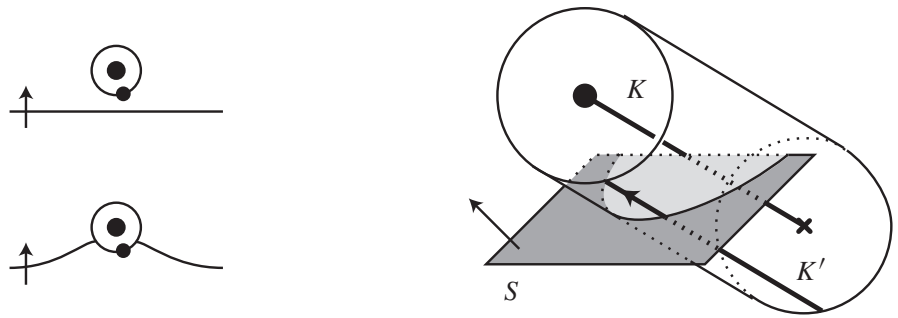

Figure 14. The intersection of $K^{\prime}$ with $S$ for the finger move depicted here is positive. The sign of the intersection switches if the central " $\bullet$ " is replaced by " $\times$ " or if the transverse orientation of $S$ is reversed.

the intersections from $A_{\mathrm{III}}$ and $A_{\mathrm{IV}}$ to the left across discs where $K$ is oriented to point into the page, and isotope the intersections from $A_{\mathrm{I}}$ to the right across a disc where $K$ is oriented to point out of the page. Figure 15 shows that moving all the intersections and standardizing the resulting pattern contributes

$$
2 f_{\mathrm{IV}}+f_{\mathrm{III}}+f_{\mathrm{I}}
$$

to the signed intersection number.
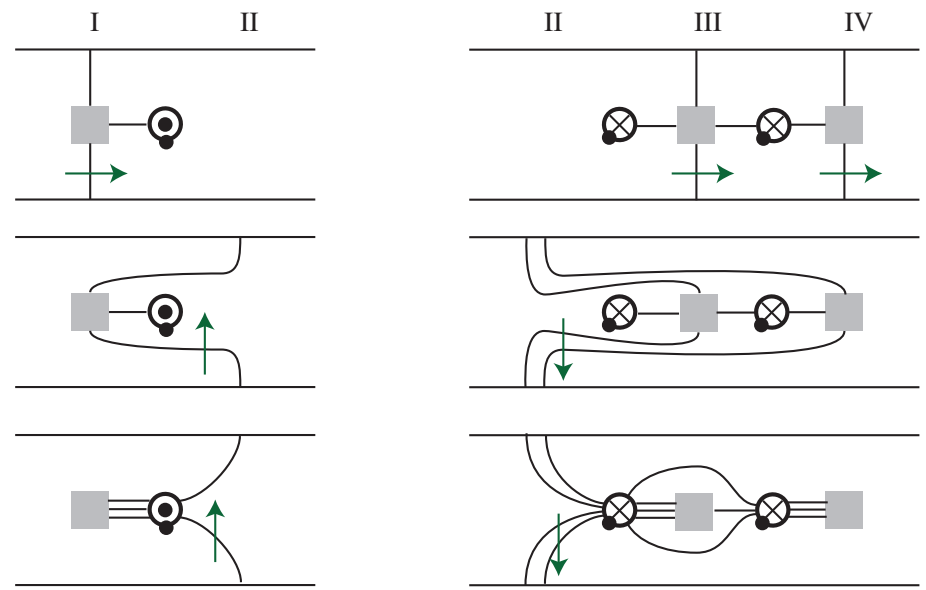

Figure 15. Isotopy and standardization: The first step slides the intersections of $A_{*}$ into $A_{\mathrm{II}}$, while the second standardizes the diagram via finger moves. In this case, $f_{*}>0$, so the contribution from regions I and III is +1 , while the contribution from region IV is +2 . If the sign of $f_{*}$ changes, so does the sign of the contribution. 

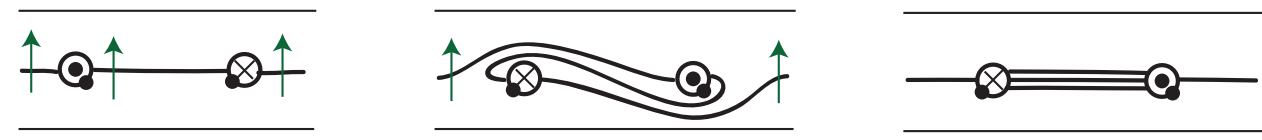

Figure 16. Standardizing after the strands of $K$ cross introduces $-m_{\mathrm{II}}+m_{\mathrm{III}}$ intersections between $K^{\prime}$ and $S$.

Performing saddle moves to eliminate all the longitudinal curves in the pattern does not change the intersection number. Furthermore, observe that the weight of the curves intersecting each side of the $N$ disc is preserved by the standardization process.

When the strands of $K$ cross, the two $N$ discs on the edges of $A_{\text {II }}$ exchange places. Standardizing the resulting pattern introduces an additional $-m_{\mathrm{II}}+m_{\mathrm{III}}=$ $-r$ intersections between $S$ and $K^{\prime}$. Summing these with the previous intersections and repeating the process at every solid torus yields the following formula for the rational Thurston-Bennequin number:

$$
\mathrm{tb}_{\mathbb{Q}}(K)=\frac{1}{r} \sum_{i}\left(-r+f_{I}^{i}+f_{\mathrm{III}}^{i}+2 f_{\mathrm{IV}}^{i}\right) .
$$

To make the formula more elegant, we repeat the same computation, but this time isotope all the intersections to $A_{\text {IV }}$ instead. Counting intersections yields:

$$
\operatorname{tb}_{\mathbb{Q}}(K)=\frac{1}{r} \sum_{i}\left(-r-f_{\mathrm{III}}^{i}-f_{I}^{i}-2 f_{\mathrm{II}}^{i}\right) .
$$

We sum the two formulae for $\operatorname{tb}_{\mathbb{Q}}(K)$ and divide by 2 , which yields the desired formula:

$$
\operatorname{tb}_{\mathbb{Q}}(K)=\frac{1}{r} \sum_{i}\left(-r-f_{\mathrm{II}}^{i}+f_{\mathrm{IV}}^{i}\right) .
$$

\section{References}

[Baker and Etnyre 2012] K. Baker and J. Etnyre, "Rational linking and contact geometry", pp. 1937 in Perspectives in analysis, geometry, and topology, edited by I. Itenberg et al., Progr. Math. 296, Birkhäuser, New York, 2012. MR 2884030

[Baker et al. 2008] K. Baker, E. Grigsby, and M. Hedden, "Grid Diagrams for lens spaces and combinatorial knot Floer homology”, 10 (2008). MR 2009h:57012 Zbl 1168.57009

[Calegari and Gordon 2009] D. Calegari and C. Gordon, "Knots with small rational genus", preprint, 2009. To appear in Comment. Math. Helv. arXiv 0912.1843

[Cornwell 2012] C. Cornwell, "Bennequin type inequalities in lens spaces", Int. Math. Res. Notices 2012:8 (2012), 1890-1916. Zbl 06034372

[Gilmer 1993] P. M. Gilmer, "Link cobordism in rational homology 3-spheres", J. Knot Theory Ramifications 2:3 (1993), 285-320. MR 94m:57012 Zbl 0797.57003 
[Hedden 2011] M. Hedden, "On the Floer homology and the Berge conjecture on knots admitting lens space surgeries”, Trans. Amer. Math. Soc. 363:2 (2011), 949-968. MR 2012f:57006 Zbl 1229.57006

[Kamishima and Tsuboi 1991] Y. Kamishima and T. Tsuboi, "CR-structures on Seifert manifolds", Invent. Math. 104:1 (1991), 149-163. MR 92c:53016 Zbl 0728.32012

[Licata and Sabloff 2010] J. Licata and J. Sabloff, "Legendrian contact homology in Seifert fibered spaces", preprint, 2010. To Appear in Quantum Topol. arXiv 1012.2421

[Lisca and Matić 2004] P. Lisca and G. Matić, "Transverse contact structures on Seifert 3-manifolds", Algebr. Geom. Topol. 4 (2004), 1125-1144. MR 2006d:57041 Zbl 1070.57013

[Massot 2008] P. Massot, “Geodesible contact structures on 3-manifolds”, Geom. Topol. 12:3 (2008), 1729-1776. MR 2009f:53139 Zbl 1152.57017

[Orlik 1972] P. Orlik, Seifert manifolds, Lecture Notes in Mathematics 291, Springer, Berlin, 1972. MR 54 \#13950 Zbl 0263.57001

[Sabloff 2003] J. M. Sabloff, "Invariants of Legendrian knots in circle bundles", Commun. Contemp. Math. 5:4 (2003), 569-627. MR 2004g:57045 Zbl 1046.57013

[Turaev 1992] V. G. Turaev, "Shadow links and face models of statistical mechanics", J. Differential Geom. 36:1 (1992), 35-74. MR 93e:57015 Zbl 0773.57012

Received August 10, 2011.

JOAN E. LICATA

MATHEMATICAL SCIENCES InSTITUTE

BUILDING 27

AUSTRALIAN NATIONAL UNIVERSITY

CANBERRA ACT 0200

Australia

joan.licata@anu.edu.au

JoShUA M. SABLOFF

DEPARTMENT OF MATHEMATICS AND STATISTICS

HAVERFORD COLLEGE

370 Lancaster AVEnUE

HAVERFORD, PA 19041

UNITED STATES

jsabloff@haverford.edu 


\title{
PACIFIC JOURNAL OF MATHEMATICS
}

\author{
http://pacificmath.org \\ Founded in 1951 by \\ E. F. Beckenbach (1906-1982) and F. Wolf (1904-1989)
}

\section{EDITORS}

V. S. Varadarajan (Managing Editor)

Department of Mathematics

University of California

Los Angeles, CA 90095-1555

pacific@math.ucla.edu

Vyjayanthi Chari

Department of Mathematics

University of California

Riverside, CA 92521-0135

chari@math.ucr.edu

\section{Robert Finn}

Department of Mathematics Stanford University

Stanford, CA 94305-2125

finn@math.stanford.edu

Kefeng Liu

Department of Mathematics

University of California

Los Angeles, CA 90095-1555

liu@math.ucla.edu
Darren Long

Department of Mathematics

University of California

Santa Barbara, CA 93106-3080

long@math.ucsb.edu

Jiang-Hua Lu

Department of Mathematics

The University of Hong Kong

Pokfulam Rd., Hong Kong jhlu@maths.hku.hk

Alexander Merkurjev

Department of Mathematics

University of California

Los Angeles, CA 90095-1555

merkurev@math.ucla.edu
Sorin Popa

Department of Mathematics University of California

Los Angeles, CA 90095-1555 popa@math.ucla.edu

Jie Qing

Department of Mathematics

University of California

Santa Cruz, CA 95064

qing@cats.ucsc.edu

Jonathan Rogawski

Department of Mathematics

University of California

Los Angeles, CA 90095-1555

jonr@math.ucla.edu

\section{PRODUCTION}

pacific@math.berkeley.edu

\section{SUPPORTING INSTITUTIONS}

ACADEMIA SINICA, TAIPEI

CALIFORNIA INST. OF TECHNOLOGY INST. DE MATEMÁTICA PURA E APLICADA KEIO UNIVERSITY

MATH. SCIENCES RESEARCH INSTITUTE NEW MEXICO STATE UNIV.

OREGON STATE UNIV.

\author{
STANFORD UNIVERSITY \\ UNIV. OF BRITISH COLUMBIA \\ UNIV. OF CALIFORNIA, BERKELEY \\ UNIV. OF CALIFORNIA, DAVIS \\ UNIV. OF CALIFORNIA, LOS ANGELES \\ UNIV. OF CALIFORNIA, RIVERSIDE \\ UNIV. OF CALIFORNIA, SAN DIEGO \\ UNIV. OF CALIF., SANTA BARBARA
}

\author{
UNIV. OF CALIF., SANTA CRUZ \\ UNIV. OF MONTANA \\ UNIV. OF OREGON \\ UNIV. OF SOUTHERN CALIFORNIA \\ UNIV. OF UTAH \\ UNIV. OF WASHINGTON \\ WASHINGTON STATE UNIVERSITY
}

These supporting institutions contribute to the cost of publication of this Journal, but they are not owners or publishers and have no responsibility for its contents or policies.

See inside back cover or pacificmath.org for submission instructions.

The subscription price for 2012 is US \$420/year for the electronic version, and \$485/year for print and electronic.

Subscriptions, requests for back issues from the last three years and changes of subscribers address should be sent to Pacific Journal of Mathematics, P.O. Box 4163, Berkeley, CA 94704-0163, U.S.A. Prior back issues are obtainable from Periodicals Service Company, 11 Main Street, Germantown, NY 12526-5635. The Pacific Journal of Mathematics is indexed by Mathematical Reviews, Zentralblatt MATH, PASCAL CNRS Index, Referativnyi Zhurnal, Current Mathematical Publications and the Science Citation Index.

The Pacific Journal of Mathematics (ISSN 0030-8730) at the University of California, c/o Department of Mathematics, 969 Evans Hall, Berkeley, CA 94720-3840, is published monthly except July and August. Periodical rate postage paid at Berkeley, CA 94704, and additional mailing offices. POSTMASTER: send address changes to Pacific Journal of Mathematics, P.O. Box 4163, Berkeley, CA 94704-0163.

PJM peer review and production are managed by EditFLOW ${ }^{\mathrm{TM}}$ from Mathematical Sciences Publishers.

PUBLISHED BY PACIFIC JOURNAL OF MATHEMATICS

at the University of California, Berkeley 94720-3840

A NON-PROFIT CORPORATION

Typeset in LATEX

Copyright $(02012$ by Pacific Journal of Mathematics 


\section{PACIFIC JOURNAL OF MATHEMATICS}

Volume $258 \quad$ No. $1 \quad$ July 2012

On the complexity of sails

LUKAS BRANTNER

Construction of Lagrangian submanifolds in $\mathbb{C P}^{n}$

QING Chen, SEN Hu and XiaOweI XU

Semisimple tunnels

SANGBUM CHO and DARRYL MCCULLOUGH

Degenerate two-boundary centralizer algebras

\section{ZAJJ DAUGHERTY}

Heegaard genera in congruence towers of hyperbolic 3-manifolds

BOGWANG JEON

The Heisenberg ultrahyperbolic equation: The basic solutions as distributions

ANTHONY C. KABLE

Rational Seifert surfaces in Seifert fibered spaces

JOAN E. LiCATA and JoshuA M. SABLOFF

Delaunay cells for arrangements of flats in hyperbolic space 